\title{
Nearly a third of tests and treatments are unnecessary: CIHI
}

- Cite as: CMAJ 2017 April 24;189:E620-1. doi: 10.1503/cmaj.1095417

anadians undergo more than a million potentially unnecessary tests and treatments each year, according to a new study by the Canadian Institute for Health Information (CIHI) and Choosing Wisely Canada (CWC), a campaign to reduce waste and avoidable harm in health care. The study of eight medical interventions found that up to $30 \%$ were performed against the recommendations of expert guidelines.

Similar rates of unnecessary care have been reported in the United States, and this is the most comprehensive report of Canadian data, says CWC chair Dr. Wendy Levinson. The notion that more is better is "deeply embedded in our culture," she said, but it shouldn't apply to medicine. Unnecessary tests and treatments expose patients to avoidable harm, increase wait times and use up scarce resources.

The study particularly highlights the risks of overmedication among seniors and children. According to $\mathrm{ClHI}$ data, one in 10 Canadian seniors regularly use benzodiazepines, even though the risks of car accidents, falls and fractures typically outweigh any potential benefits. Doctors commonly prescribe the sedatives for insomnia. But Levinson noted there are "other ways to manage sleep problems that are safer." These include stopping naps, shutting off electronics before bed and short-term use of melatonin supplements.

The study also showed that doctors are increasingly and inappropriately prescribing antipsychotics to children and youth. Between 2005 and 2012, Manitoba, Saskatchewan and British Columbia saw a

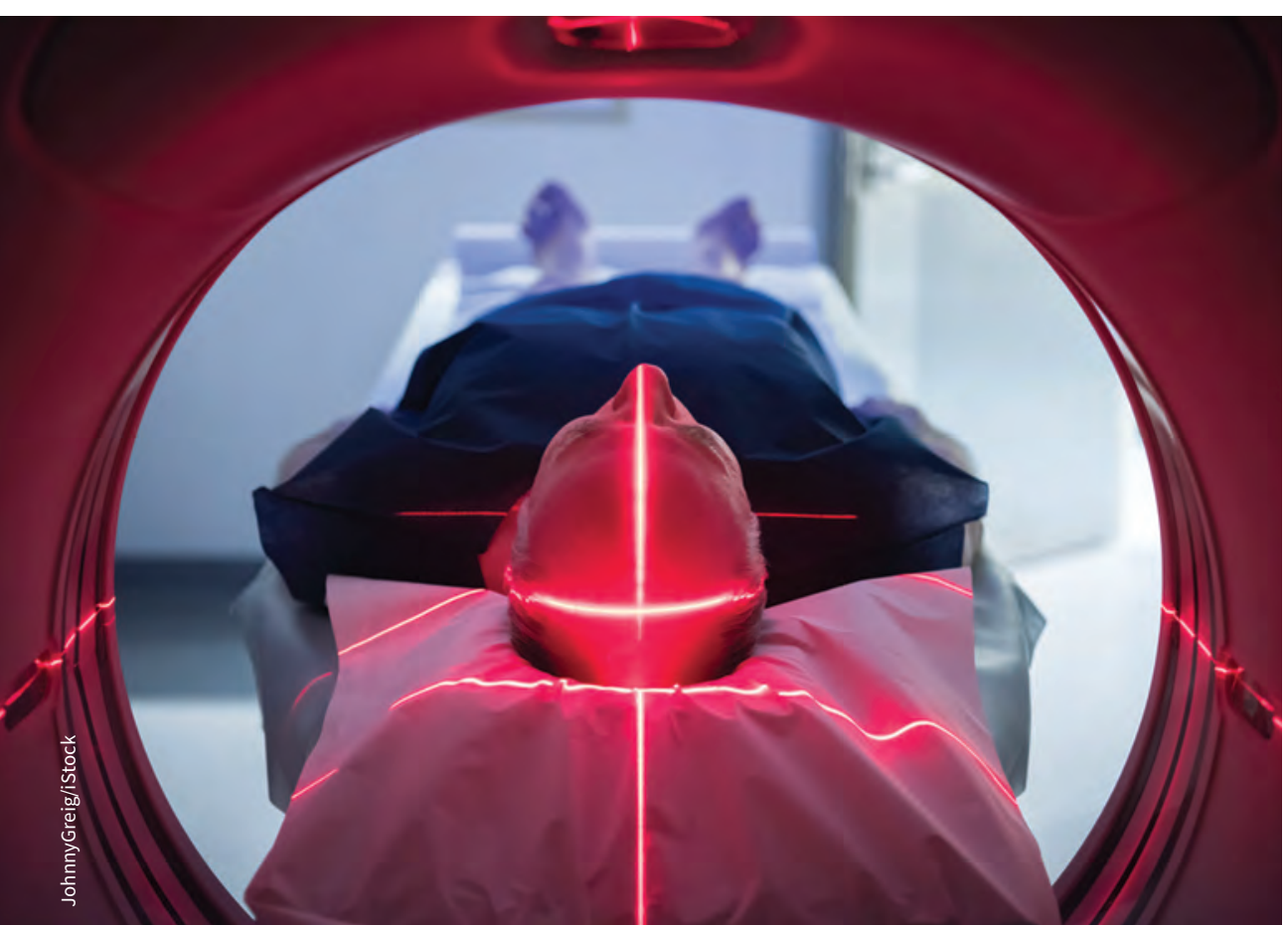

A new study sheds light on Canada's problem of "more is better" medicine.

$300 \%$ increase in dispensing of quetiapine to young people aged five to 24 , even though the drug is not recommended for use in children and youth.

When it came to imaging and screening, the study found that routine often trumped recommendations.

One in three low-risk patients with minor head trauma in Ontario and Alberta had a computerized tomography (CT) scan, even though most patients won't have a serious brain injury and the scans won't improve their outcomes.

In Alberta, $30 \%$ of patients with lowerback pain, but no red flags of a more serious condition, had at least one x-ray, CT or magnetic resonance imaging (MRI) scan, even though the pain usually clears up over time without intervention.

Meanwhile, $18 \%-35 \%$ of patients undergoing low-risk surgery in Ontario, Saskatchewan and Alberta had some kind of preoperative test, which is unnecessary and can end up delaying surgery.

In all of these cases, the risks of harm from radiation and false-positive results leading to more unnecessary care outweigh the chance of catching a hidden problem.

There were some positive trends, however. Red blood cell transfusions for elective hip and knee replacements have decreased in recent years, although the transfusion rates across facilities ranges from $0 \%$ to $33 \%$. Wide variation was also reported across other interventions.

Levinson argued this is good news because it signals it's possible to reduce rates of unnecessary care. Variations in practice can also point to the factors influencing some physicians to provide more tests and treatments. For example, ClHI's analysis of facilities in Alberta and Saskatchewan found that physicians who performed fewer surger- 
ies tended to order more preoperative tests, suggesting confidence or training issues.

Other data showed that emergency departments with high trauma volumes had higher percentages of potentially unnecessary head scans. This may indicate there are more diagnostic imaging machines on site, which could contribute to the decision by some physicians to order a scan.

"For us doctors, we don't want to be seen as the guilty party," admitted Dr. Lau- rent Marcoux, Canadian Medical Association president-elect. "The patient is involved, the system is involved." Ultimately, however, "we have a responsibility," he said.

Changing the culture of medicine will require education for both physicians and patients, Marcoux added. "I'm very confident the young doctors in Canada are more aware than ever about the problem of over-diagnosis."

Levinson said that education starts in conversations between doctors and patients. "When physicians listen and deeply understand the patient's concerns and they have a discussion about the pros and cons, the patient feels reassured."

Some physicians worry it will take longer to discuss a test than to order it. But often a quick conversation upfront "prevents longer conversations or return visits later," Levinson explained. "Our research shows it takes one minute on average."

Lauren Vogel, CMAJ

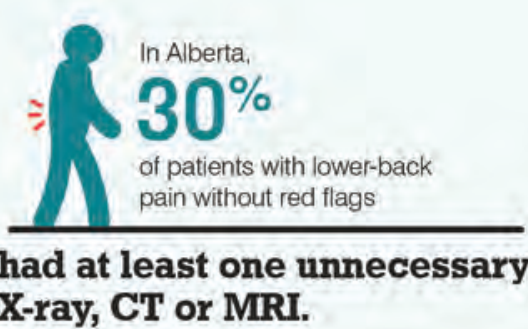

X-ray, CT or MRI.

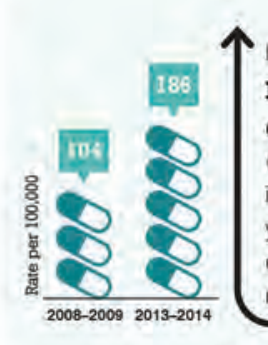

In Manitoba, Saskatchewan and B.C rates of low-dose quetiapine (commonly used to treat insomnia) increased among children and young adults age 5 to 24 even though this is not recommended by experts.

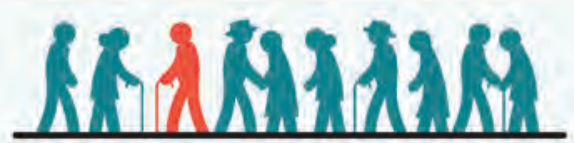

1 in 10 seniors in Canada uses a benzodiazepine (sedative-hypnotic) on a regular basis, even though this is not recommended by experts.

\section{In Ontario, Saskatchewan and Alberta,}

$18 \%$ to $35 \%$

of patients who had a low-risk procedure had a preoperative test.

\begin{tabular}{l|l|l|l|l}
\hline EMERGENCY \\
$\begin{array}{l}\text { Alberta with low-risk minor head trauma } \\
\text { received a CT head scan. }\end{array}$
\end{tabular}

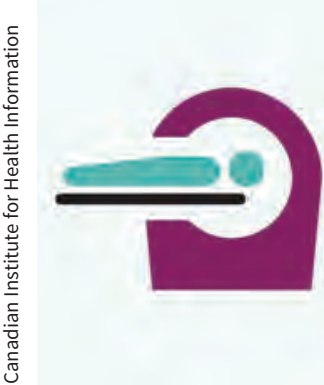

\section{In Ontario}

$23 \%$

of inpatients with delirium

had a potentially unnecessary head CT scan.

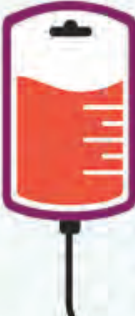

Red blood cell

transfusions for elective hip $(12 \%)$ and knee $(8 \%)$ replacements have decreased but continue to be done across Canada, even though blood is a precious resource. 